\title{
Ecological and evolutionary adaptations shape the gut microbiome of BaAka African rainforest hunter-gatherers
}

Andres Gomez ${ }^{1,2,+}$; Klara Petrzelkova ${ }^{3,4,5,6}$; Carl J. Yeoman ${ }^{7}$; Michael B. Burns ${ }^{1,2}$; Katherine, R. Amato ${ }^{8}$; Klara Vlckova ${ }^{4}$; David Modry ${ }^{4,9}$; Angelique Todd ${ }^{10}$; Carolyn A. Jost Robinson $^{11}$; Melissa J. Remis ${ }^{12}$; Manolito Torralba ${ }^{13}$; Karen E. Nelson ${ }^{13}$; Franck Carbonero $^{14,15,16}$; H. Rex Gaskins ${ }^{14,15}$; Brenda Wilson ${ }^{14,17}$; Rebecca M. Stumpf ${ }^{14,18}$; Bryan A. White ${ }^{14,15}$; Steven R. Leigh ${ }^{8,14,18,+, *}$ and Ran Blekhman ${ }^{1,2,+*}$

${ }^{1}$ Department of Genetics, Cell Biology, and Development, University of Minnesota, Twin Cities, MN, 55108

${ }^{2}$ Department of Ecology, Evolution and Behavior, University of Minnesota, Twin Cities, MN, 55108

${ }^{3}$ Institute of Vertebrate Biology, Academy of Sciences of the Czech Republic, Brno, Czech Republic

${ }^{4}$ Department of Pathology and Parasitology, Faculty of Veterinary Medicine, University of Veterinary and Pharmaceutical Sciences, Brno, Czech Republic

${ }^{5}$ Institute of Parasitology, Biology Center of the Academy of Sciences of the Czech Republic, České Budějovice, Czech Republic

${ }^{6}$ Liberec Zoo, Liberec, Czech Republic

${ }^{7}$ Department of Animal and Range Sciences, Montana State University, Bozeman, MT, 59717

${ }^{8}$ Department of Anthropology, University of Colorado, Boulder, CO, 80309, USA

${ }^{9}$ CEITEC, Central European Institute for Technology, Brno, Czech Republic

${ }^{10}$ World Wildlife Fund, Dzanga-Sangha Protected Areas, Bayanga, Central African

Republic

${ }^{11}$ Department of Anthropology, University of North Carolina, Wilmington, NC, 28403

${ }^{12}$ Deptartment of Anthropology, Purdue University, West Lafayette, IN, 47907

${ }^{13}$ The J. Craig Venter Institute, Rockville, MD, 20850

${ }^{14}$ Institute for Genomic Biology, University of Illinois at Urbana Champaign, IL, 61801

${ }^{15}$ Department of Animal Sciences, University of Illinois at Urbana Champaign, IL, 61801

${ }^{16}$ Department of Food Science, University of Arkansas, Fayetteville, AK, 72704

${ }^{17}$ Department of Microbiology, University of Illinois at Urbana-Champaign, IL, 61801

${ }^{18}$ Department of Anthropology, University of Illinois at Urbana Champaign, IL, 61801

${ }^{+}$Corresponding authors: email: gomeza@umn.edu (AMG); Steven.Leigh @ colorado.edu (SRL); blekhman@umn.edu (RB)

*These authors contributed equally to this work

Classification: BIOLOGICAL SCIENCES: Anthropology; Ecology; Evolution; Microbiology 


\begin{abstract}
The gut microbiome provides access to otherwise unavailable metabolic and immune functions, likely affecting mammalian fitness and evolution. To investigate how this microbial ecosystem impacts evolutionary adaptation of humans to particular habitats, we

5 explore the gut microbiome and metabolome of the BaAka rainforest hunter-gatherers from Central Africa. The data demonstrate that the BaAka harbor a colonic ecosystem dominated by Prevotellaceae and other taxa likely related to an increased capacity to metabolize plant structural polysaccharides, phenolics, and lipids. A comparative analysis shows that the BaAka gut microbiome shares similar patterns with that of the Hadza,

10 another hunter-gatherer population from Tanzania. Nevertheless, the BaAka harbor significantly higher bacterial diversity and pathogen load compared to the Hadza, as well as other Western populations. We show that the traits unique to the BaAka microbiome and metabolome likely reflect adaptations to hunter-gatherer lifestyles and particular subsistence patterns. We hypothesize that the observed increase in microbial diversity

15 and potential pathogenicity in the BaAka microbiome has been facilitated by evolutionary adaptations in immunity genes, resulting in a more tolerant immune system.
\end{abstract} Keywords: BaAka hunter-gatherers; Pygmy; gut microbiome; adaptation; evolution

\title{
Significance
}

Human ecological adaptation requires changes at the genomic level. However, the gut

20 microbiome, the collection of microbes inhabiting the gastrointestinal tract and their functions, also responds significantly to ecological challenge. To determine how the gut microbiome responds to evolutionary adaptations in the host, we profiled gut bacterial communities of the BaAka, rainforest hunter-gatherers from Central Africa. The gut 
microbiome of the BaAka shows adaptations to metabolize foods rich in fiber, tannins

25 and fats. Similarly, higher bacterial diversity and abundance of pathogenic bacteria, compared to other hunter-gatherers and western populations, suggest that the BaAka

immune system evolved to coexist with increased pathogen threats. Accordingly, these results show how the gut microbiome contributes to human ecological plasticity, impacting host adaptation and evolution.

30 


\section{Vbody}

Particular gut microbiome patterns have been linked to specific physiological phenotypes in humans $(1,2)$. Recently, the characterization of gut microbiomes across

50 geographically and culturally diverse human populations have helped researchers pinpoint the effects of lifestyle factors on the composition of these microbial communities and the potential influence of gut microbes in human health and disease (35). For instance, specific gut microbiome assemblages, likely supported by westernized diets and industrialized lifestyles, are believed to play a role in the high incidence of

55 metabolic disorders observed in some modern human populations (6). These patterns are opposed to the more ancestral microbiome traits seen in traditional societies (4). Thus, comparative analyses of diet-microbiome interactions facilitate an understanding of diverse lifestyles and diets on human adaptation and health.

With respect to population-specific adaptations, persistent debate has accompanied

60 the question of whether or not forest foraging lifestyles among Central African rainforest hunter-gatherer populations supply adequate nutrition, in the context of specific phenotypes and ecological challenges (7-9). In this regard, small human body size, a characteristic common to some rainforest hunter-gatherer populations (the "pygmy" phenotype), has been variably hypothesized to have evolved in response to energetic

65 limitations, forest density, high heat and humidity, increased parasite load and high adult mortality (10-12). Given the role of the gut microbiome in harvesting nutrients from low energy foods and their critical impact in immune balance, understanding the gut microbiome of these hunter-gatherer populations may provide insights into their ecology and evolutionary adaptations. 
70 Here, we explore gut microbiome and metabolome composition in stool samples of the BaAka, forest foragers from the Dzanga Sangha Protected Areas (DSPA), Central African Republic. A comparative analysis of microbiome composition was conducted, including the Hadza hunter-gatherers from Tanzania, urban living Italians (13) and US Americans (2). Thus, we predict that the characterization bacterial communities and

75 metabolomes in the BaAka gut contributes to persistently debated anatomical, genetic, and physiological specializations that help distinguish forager populations from nontraditional western societies.

\section{Results}

The BaAka gut microbiome. Gut microbiome composition was profiled through

80 454-16S rRNA pyrosequencing of stool samples from 27 BaAka. After quality control, we obtained a total of 151,407 pyrotag reads, with an average number of $5,607 \pm 1,841$ reads per sample (range 2,939-10,673). Firmicutes and Bacteroidetes dominated the BaAka gut microbiome (45 \pm 16.9 and $39 \pm 20.6 \%$ respectively) (Figure 1a).

Unclassified bacteria comprised $10.8 \pm 14.4 \%$ of all reads, whereas Proteobacteria and

85 Spirochaetes were the most abundant among minor phyla $(2.3 \pm 2.9$ and $1.6 \pm 2.2 \%$ respectively).

A taxonomic analysis at family level detected 55 taxa in the BaAka microbiome, with Prevotellaceae (mainly Prevotella spp.) identified as the predominant family $(33.6 \pm 23.2$ \%) (Figure 1a). High abundances of Prevotellaceae (up to 53\% in average) are also

90 predominant in other African populations that rely on high-fiber content diets $(14,15)$. Together, members of the Clostridiales order (Lachnospiraceae (13.31 $\pm 8.49 \%$ ), Ruminococcaceae $(13 \pm 8 \%)$, Clostridiaceae $(8.43 \pm 9.35 \%)$, and unclassified 
Clostridiales $(2.79 \pm 2.27 \%))$ comprised the second most abundant taxa $(37.5 \pm 28.6 \%)$, while other members of the Bacteroidetes (mainly unclassified Bacteroidales) reached

$953.51 \pm 4.5 \%$ relative abundance. Spirochaetaceae (primarily Treponema spp.) was the most abundant among minor taxa comprising $1.6 \pm 2.2 \%$ in the BaAka.

The BaAka gut metabolome and microbiome-metabolome interactions. From the 27 samples analyzed, 20 yielded usable gas chromatography/mass spectrometry (GC/MS) spectra. Analysis of both polar and non-polar metabolites in these stool samples detected

100 a total of $~ 2,500$ mass spectral features, and from these, 260 compounds were positively identified. After eliminating metabolites found in less than $50 \%$ of samples, the stool metabolomes of the BaAka were mainly grouped into lipids (40.8\%), carbohydrates (19\%), sterols (18.9\%), phosphates (8\%), organic acids (7.9\%), amino acids and amines (3.3\%), and bile acids (1.7\%) (Figure 1b). Coprostan-3-ol, the main conversion product

105 from cholesterol metabolism in the distal colon (16), was the most abundant metabolite $(15.6 \pm 10.6 \%)$, followed by glycerol $(12.7 \pm 11.74 \%)$, stearic acid $(11.9 \pm 8.7 \%)$ palmitic acid $(10 \pm 6.5 \%)$, phosphoric acid $(8 \pm 8.5 \%)$ and lactic acid $(4.5 \pm 5 \%)$. Other metabolites comprised $2 \%$ of abundance or less (Figure 1b).

Spearman correlations between metabolites (relative abundance) and taxa (at family

110 level) exhibiting $r h o \geq 0.5$ (false discovery rate, $q<0.05$ ) (Table S1) were chosen to visualize microbiome-metabolite interactions and infer potential microbiome functions (Figure 1c). The most dominant taxa in the BaAka gut established correlations with abundances of metabolites involved in plant cell wall processing and phenolics. For instance, Prevotellaceae formed the strongest associations with sorbitol, a sugar alcohol 115 and a product of glucose reduction and with keto-L-gluconic acid, another product of 
glucose metabolism. Abundances of Lachnospiraceae correlated with arabinose and rhamnose, structural components of pectic polysaccharides and hemicelluloses, and with propanoic acid, a product of polysaccharide fermentation. Ruminococcaceae showed strong associations with ethanolamine and pyruvic acid, both metabolites derived from

120 energetic turnover mechanisms, and with benzeneacetic acid, an aromatic compound likely associated to the phenolic fraction of plants (17). The same is the case for Spirochaetaceae, which formed associations with methyl-phenol. In this regard, quinic acid, also a plant polyphenolic, formed several strong connections with unclassified Clostridiales and Bacteroidetes, and also with Victivalllaceae, and Anaeroplasmataceae,

125 both of which have been related to increased plant matter degradation in the herbivore gut $(18,19)$. Other abundant taxa in the BaAka such as Clostridiaceae and Lactobacillaceae also formed strong associations with palmitic acid, deoxycholic acid (a secondary bile acid) and with other metabolites associated with lipid processing (Figure 1c).

\section{The BaAka gut microbiome in comparison to that of other hunter-gatherer and}

130 non-western societies. To understand the composition of the BaAka gut microbiome in the context of other populations and diets, we incorporated data from another huntergatherer group, the Hadza from Tanzania, and two western populations, Italians and US Americans. These data indicate that the BaAka gut microbiome exhibits significantly higher richness and diversity ( $\mathrm{P}<0.001$, Wilcoxon rank-sum test) (Figure 2a) and less

135 inter-individual variation than those of the Hadza hunter-gatherers from Tanzania, Italians, and Americans from the US (Figure 2b; Permutation test for difference in medians, $\mathrm{P}<0.001)$. 
Although the gut microbiomes of the BaAka were more similar to those of the Hadza than they were to those of the western groups (Figures $\mathbf{2 b}, \mathbf{2 c}$ and S1), some taxa were

140 significantly more abundant only in the BaAka (FDR-adjusted Kruskal-Wallis tests, $\mathrm{q}<0.01)$. This is the case of Clostridiaceae, Anaeroplasmataceae, and Victivallaceae (Figures 3, S2a, S2b, and table S2). Ribosomal sequences of these taxa were all related to bacteria with increased capacity for metabolizing plant glycosides (18-20). Unclassified sequences affiliated to the Verrucomicrobia and Alphaproteobacteria; also

145 increased only in the BaAka, have been previously reported as abundant in stool samples of mountain gorillas, which rely heavily on high fiber diets (21), and in the gut microbiome of children from Bangladesh, whose diet is high in plant polysaccharides (22) (Figure S2 and table S1).

Other families such as Mogibacteriaceae, Corynebacteriaceae, Coriobacteriaceae, and 150 Peptostreptococcaceae were also significantly more abundant in the BaAka only. Although these taxa can be commonly found in the mammalian gut, they all have also been associated to potential inflammation or infection (23-26). Taxa related to Odoribacteraceae were completely absent in the BaAka compared to all other groups. Among the taxa that were significantly more enriched in both African groups (BaAka 155 and Hadza) than in westerners (FDR-adjusted Kruskal-Wallis tests, q<0.01) (Figures 3, S2 and Table S1) we found Prevotellaceae, Paraprevotellaceae, Spirochaetaceae, unclassified Bacteroidales, and Succinivibrionaceae. These taxa appear to be consistently enriched in other African foragers and farmers, as well in other non-western traditional societies $(3,5,13,14)$. 
160 Bacteroidaceae (mainly Bacteroides), Porphyromonadaceae, Barnesiellaceae and Rikenellaceae were more abundant in Americans and Italians (FDR-adjusted KruskalWallis tests, $\mathrm{q}<0.01$ ) compared to the African groups (Figures 3, S2 and Table S1). These closely related taxa (27) are believed to be signatures of western gut microbiomes $(28,29)$. Bifidobacteriaceae was significantly more enriched in Italians and almost absent 165 in all other groups (FDR-adjusted Kruskal-Wallis tests, $q<0.01$ ) (Figures 3, S2 and

\section{Table S1).}

Microbiome functional comparison across populations. To predict the potential functional roles played by gut bacterial communities in the BaAka compared to all other groups and in both African hunter-gatherers vs. westerners, we used the PICRUSt

170 (Phylogenetic investigation of communities by reconstruction of unobserved states) open source software (30). Increased predicted abundance of genes involved in infection pathways (Staphylococcus aureus and Vibrio cholerae) distinguished the BaAka over all other groups (FDR-adjusted Kruskal-Wallis tests, q<0.01) (Figure 4). Functional predictions also revealed that the gut microbiome of both western groups were

175 significantly more enriched in genes involved in carbohydrate metabolism; galactose, starch and sucrose and the pentose phosphate pathway compared with the Hadza and the BaAka (FDR-adjusted Kruskal-Wallis tests, $\mathrm{q}<0.01)$.

\section{Discussion}

The present data indicate that the intestinal microbiome of the BaAka foragers may

180 harbor compositional and functional features that likely reflect their subsistence, lifestyle, and ecology. In addition, through comparisons with the Hadza hunter-gatherers, US Americans and Italians, the data show microbiome patterns that distinguish traditional 
foragers from western societies. Three main features seem to characterize the gut microbiome of the BaAka hunter-gatherers, an increased capacity for processing

185 structural polysaccharides and phenolics, a metabolome dominated by lipid-derived markers and high gut bacterial diversity, including prevalence of potential pathogens.

\section{The BaAka gut microbiome shows adaptations for processing phenolic-rich}

fibrous foods. The observation of a colonic ecosystem adapted to break down fiber may be related to the important role of fibrous polysaccharides such as bitter manioc root,

190 yams, leaves (Gnetum Africanum) and wild fruits in the BaAka diet (31). Starches and wild leaves and fruits (rich in fiber and phenolics (32)) seem to be staple foods for hunter-gatherers in tropical rainforests $(33,34)$ and for the BaAka in Central African Republic and southeast Congo $(31,35)$.

Along these lines, the dominance of Prevotellaceae in the BaAka gut may be

195 associated with large influx of xylane, pectin, and starch into their colonic ecosystem $(36,37)$. High abundances of Prevotellaceae, which were also shared with the Hadza, have also been observed in the gut microbiome of other rural farming groups in Africa and South America, who depend on fibrous starches for subsistence (3-5, 13-15). Prevalence of Clostridiaceae, Ruminococcaceae and Lachnospiraceae and the

200 correlations seen between these taxa and plant polyphenolics such as benzenes, protocatechuic acid, quinic acid and other metabolites derived from fiber processing, might further support an increased capacity to break down phenolic-rich fibers in the BaAka (38).

The shared patterns seen between the BaAka and Hadza, in comparison to those in 205 US Americans and Italians demonstrate a distinctive non-western gut microbiome. This 
observation is supported by dominance of other potentially fibrolytic taxa such as Succinivibrionaceae (39) and Spirocahetaceae (who was also significantly associated to abundances of phenolics) in the 2 hunter-gatherer groups. Interestingly, prevalence of Spirochaetaceae has not only been seen in the gut microbiome of other rural and

210 traditional societies worldwide $(3-5,13,22)$, but also, along with Prevotellaceae, in coprolites from extinct humans in northern Mexico (1,400 years B.P) (40).

Thus, Prevotellaceae and Spirochaetaceae may represent more ancestral gut microbiome states, specifically adapted to fiber processing. Moreover, these two taxa have also been consistently found in lowland and mountain gorillas as well as in

215 chimpanzees $(21,41,42)$, humans' closest evolutionary relatives. The fact that these taxa are notably low in abundance or absent in westerners $(4,5)$, implies that large-scale agriculture and industrialization has caused loss of fiber processing diversity in modern microbiomes in favor of taxa likely specialized in metabolizing more refined carbohydrates. This is supported by the higher abundance of predicted carbohydrate

220 processing metabolic pathways (starch, sucrose and galactose) and other closely related taxa from the Bacteroidales such as Barnesiellaceae, Porphyromonadaceae and Rikenellaceae in Italians and US Americans.

The BaAka metabolome shows high lipid processing activity. It is notable that fatty acids and metabolites derived from lipid processing were the predominant

225 metabolites in the BaAka. Thus, the BaAka may incorporate significant amounts fat in their diet, probably from wild meat, fish or lipid-rich nuts. Indeed, nuts from Irvingia wombolu constitute one of the main staple foods for the BaAka (31). These foods are richer in polyunsaturated and monounsaturated fats, compared to the predominantly 
saturated fats found in raised cattle (43). The combination of these unsaturated fats along

230 with plant foods rich in fiber and polyphenolics (reported to have hypolipidemic and antioxidant effects (44)) are believed to be associated with the lower incidence of western-like metabolic diseases in hunter-gatherers (45). Furthermore, consistent with the microbiome-metabolome interaction network presented herein, colonic bacteria also interact with fatty acids to mediate conversion of other metabolites with potential

235 antioxidant properties, such as conjugated linoleic acid (C18:2) and stearic acid (C18:0) (46), the most abundant fatty acids in the BaAka metabolome.

These metabolome-microbiome markers also motivate questions regarding energy storage and expenditure patterns in the BaAka. Metabolomes high in long-chain fatty acids and cholesterol derived markers as well as interactions of these metabolites with

240 abundances of Lacobacillaceae have also been observed in mice and humans under high fat and high-energy diets (47). Thus, although energy intake has been suggested to be low in the wider BaAka community (35), which may result from limited food access $(10,48)$, these data indicate that the BaAka may not be energetically deficient and that their gut microbiomes may contribute significantly to energy harvest. However, it is

245 unclear whether these patterns are exclusive of this group of individuals or if these observations are applicable to a wider BaAka population in light of strong fluctuations in resource availability they experience (31).

The BaAka gut microbiome shows increased diversity and prevalence of potentially pathogenic bacteria. Even though the gut microbiome of the BaAka shares

250 metabolic adaptations to increased fiber processing with other non-western traditional groups, these foragers show significantly higher bacterial diversity and abundance of 
potential pathogens compared with the Hadza, Italians and US Americans. Increased bacterial diversity in the colonic ecosystem has generally been related to dietary diversity and higher fiber intakes (49). However, the Hadza, who show significantly lower

255 bacterial diversity compared to the BaAka, also exhibit these dietary patterns (13). Thus, another possible explanation for the observed increase in bacterial diversity in the BaAka over all other groups could be related to immunological benefits and adaptive tools to counteract potential pathogen threats (50). In this regard, increased parasite loads and infectious diseases are more common in populations inhabiting tropical rain forest (51).

260 This may be the case of the BaAka $(10,12)$, who could benefit from the completive exclusion mechanisms offered by a rich bacterial ecosystem. In deed, long-term monitoring of gastrointestinal parasites in these BaAka revealed that most of them suffer from multiple infections of pathogenic gastrointestinal parasites, i.e. Trichuris trichuira, Necator spp,, Ascaris lumbricoides, Strongyloides spp., in many cases in high intensities

265 (Petrzelkova at al., unpublished data). Nevertheless, it remains to be proven whether the savanna-woodland habitats occupied by the Hadza, which make them less susceptible to gastrointestinal pathogens (52) also make them less dependent on higher bacterial diversity to neutralize pathogens compared with the BaAka.

Notably, genetic variation data indicate evolutionary adaptations in genes causing 270 negative regulation of cytokine signaling and hence, potentially increased susceptibility to infection in the BaAka (53). Such data have not ben reported for the Hadza. This phenomenon may favor a more tolerant mucosal immune system, which may correlate with the observed increased microbiome richness, pathogen prevalence and predicted abundance of bacterial infection genes. Thus, further research should focus on whether 
275 high abundance of potential pathogens in the BaAka and a highly diverse gut microbiome are related to long-term, co-evolutionary host-microbe interactions, favored by more tolerant immune mechanisms (54).

That the gut microbiomes of the BaAka were significantly less variable than those of all other groups may also reflect strong selection pressures shaping their gut bacterial

280 communities. Although still controversial, natural selection in West African foragers like the BaAka is believed to have favored genomic signatures involved in growth hormones (55), shorter life span and immunity (53). These may be important factors behind their small body size and high pathogen loads $(10,48)$. If these traits evolved in response to the ecological challenges faced by the BaAka in a dense, humid, resource-limited, and 285 pathogen-prone tropical forest, it is likely that low inter-individual variability in gut microbiomes, high bacterial diversity and the microbiome-metabolome traits presented here are also adaptations providing the BaAka with key nutritional and immunological adaptive tools.

Concluding remarks and future perspectives. Besides microbiome and

290 metabolomes adaptations to particular ecological and lifestyle patterns in the BaAka, we have presented further evidence of a distinctive non-western gut microbiome, likely compatible with more ancestral human states. However, questions remain; although our data show that the BaAka microbiome is potentially active in fiber processing and energy harvesting, these results should be tested over a wider sample size, including different

295 BaAka populations under dietary fluctuations and their Bantu neighbors at Dzanga. In this regard, the microbiome similarities seen between the BaAka and traditional African, South-American and Asian societies suggest that metabolic adaptations to phenolic and 
fiber-rich foods is not exclusive of the BaAka and that it may be a trait of traditional nonwestern groups. Also, the existence of an actual "ancestral" gut microbiome,

300 characterizing these traditional non-western societies requires further exploration. This issue may be better understood by increasing efforts to reconstruct the vast uncharacterized diversity in these human populations and with comparative analyses that include wild nonhuman primates (56). Likewise, we urge the need to describe molecular interactions between diets, the gut microbiome and host immune markers in hunter-

305 gatherers and industrialized societies. These approaches have the potential to unravel the benefits of increased gut bacterial diversity in traditional societies and may help us understand the forces that shaped modern microbiomes in the context of westernized disease patterns.

\section{Methods}

310 Study site, subjects and sample collection. Stool samples were collected from 27 BaAka. The group included 20 BaAka partially hired as gorilla trackers by the Primate Habituation Program (PHP) in DSPA and 7 female partners. The trackers alternate periods of time between the research camps, tracking the gorillas in the Park and their villages. Both trackers and their partners frequently do either day hunts from the villages

315 or longer hunting trips to the Special Reserve. The samples were collected from JuneAugust of 2011 upon consent from all individuals, and also as part of an effort to characterize their parasite loads. Approximately $1 \mathrm{~g}$ of feces was collected in 2-ml Eppendorf tubes containing RNAlater (Invitrogen, life technologies). Samples were kept at room temperature for a maximum of one month before transport to the Institute of 320 Vertebrate Biology, Academy of Sciences of the Czech Republic, where they were kept 
at $-20^{\circ} \mathrm{C}$ until they were shipped to the University of Illinois at Urbana-Champaign, USA where DNA was extracted. All work was approved according to rules and regulations from the Ministre de l'Education Nationale, de l'Alphabetisation, de l'Enseignement Superieur, et de la Recherche (Central African Republic) as well as the Institutional

325 review board for the protection of human subjects from the University of Illinois at Urbana-Champaign, permit number 13045, September 4, 2014.

Sample processing and DNA analyses. DNA was extracted from stool samples using the MoBio Ultraclean Soil kit (MoBio Laboratories Inc., Carlsbad, CA, USA). The V1-V3 region of the $16 \mathrm{~S}$ rRNA gene was PCR amplified (20 cycles: at $94^{\circ} \mathrm{C}$ for 30 s, at

$33048^{\circ} \mathrm{C}$ for $30 \mathrm{~s}$, and at $72^{\circ} \mathrm{C}$ for $\left.2 \mathrm{~min}\right)$ using primers $27 \mathrm{f}\left(5^{\prime}-\right.$

AGAGTTTGATYMTGGCTCAG-3', corresponding to nucleotides 8-27 of the Escherichia coli 16S rRNA gene) and 534r (5'- ATTACCGCGGCTGCTGGCA-3', tagged with identifying barcodes, MID tag 1-50). The amplicons were multiplexed and pyrosequenced using 454 FLX-Titanium technology at the J. Craig Venter Institute

335 (Rockville, MD, USA). After removing low quality sequences (<Q30, sequences shorter than $250 \square$ nt, sequences with homopolymers longer than six nucleotides, and sequences containing ambiguous base calls or incorrect primer sequences) with custom-perl scripts, reads were processed using the online tool mothur, which included denoising, chimera check and denovo clustering of sequences sharing $\geq 97 \% 16$ S rRNA sequence identity,

340 into an operational taxonomic unit (OTU) (57).Taxonomic profiles of sequences was also conducted at genus, family, order, class and phylum level using the Ribosomal Database Project (RDP) Classifier (58) and the phylotype function within mothur. After sequence processing, OTUs detected fewer than five times across the entire data set and/or in fewer 
than 3 individuals were removed to avoid including probable sequence artifacts. For

345 comparisons with the Hadza, Italians and Americans, we downloaded sequences deposited in MG-RAST, project ID 7058 (13), and from a subset of data available in the Human microbiome project (HMP) (http://www.hmpdacc.org/). 16S rRNA Sequences from Italians and the Hadza correspond to the V4 variable region obtained with 454 pyrosequencing. Sequences from US Americans, part of the HMP, correspond to the V1-

350 V3 16SrRNA variable region, also obtain through 454 pyrosequencing. Thus, all sequence reads for the intergroup comparisons were analyzed using the closed reference OTU picking script against the greengenes database, as implemented in the QIIME pipeline (59). This pipeline was also used to calculate phylogenetic diversity as well as UniFrac distances. The PICRUSt (Phylogenetic investigation of communities by

355 reconstruction of unobserved states) open source software (30) was used to predict the potential functional roles played by gut bacterial communities in the four groups. Sequence data from the BaAka have been deposited in MG-RAST, project ID XXXX. Metabolomic analyses. Extraction for polar and non-polar metabolites was performed separately. Metabolites were extracted with $1 \mathrm{ml}$ of $70 \%$ methanol and 360 sonication in QSonica Microson XL2000 Ultrasonic Homogenizer (QSonica, LLC., CT, USA). Lysed cell pellets were subsequently fractionated at room temperature with $5 \mathrm{~mL}$ of $70 \%$ methanol, and chloroform, accompanied by centrifugation (10 min at maximum speed). One milliliter of each extract was evaporated under vacuum at $-60^{\circ} \mathrm{C}$ and then dried extracts were derivatized (See supplemental note for details). The spectra of all 365 chromatogram peaks were compared with electron impact mass spectrum libraries NIST08 (NIST, MD, USA), W8N08 (Palisade Corporation, NY, USA), and a custom- 
built library of 520 unique metabolites. All known artificial peaks were identified and removed. To allow comparison between samples, all data were normalized to the internal standard in each chromatogram and the sample dry weight (DW). The spectra of all

370 chromatogram peaks were evaluated using the AMDIS 2.71 (NIST, Gaithersburg, MD, USA) program. Metabolite concentrations are reported as "(analyte concentration relative to hentriacontanoic acid) per gram dry weight" (relative concentration), i.e., as target compound peak area divided by the internal standard (IS) peak area (IS concentration is the same in all samples): $\mathrm{N}_{\mathrm{i}}=\mathrm{X}_{\mathrm{i}} \times \mathrm{X}^{-1}$ IS $\times \mathrm{g}$ wet $\mathrm{w}$.

375 Statistical analyses. All multivariate and community analyses were conducted using the $c a$, vegan and labdsv packages of $\mathrm{R}(60)$ on the relative abundance of each taxon (6163). Indicator species analysis (64) was used to characterize the most prominent taxa in the gut microbiome of each group. Spearman correlations and Kruskal-Wallis tests were completed using the psych and pgirmess packages of $\mathrm{R}(65,66)$. GC/MS spectra data

380 from metabolomic analyses were transformed as follows: i) metabolites with $>50 \%$ of missing data were removed from the set and ii) the relative abundance of a given metabolite was expressed relative to the sum of all the spectra obtained for a given sample.

\section{Acknowledgements}

385 We would like to thank the government of the Central African Republic, namely the Ministre de l'Education Nationale, de l'Alphabetisation, de l'Enseignement Superieur, et de la Recherche for providing research permits to conduct our work in the Central African Republic; World Wildlife Fund and the administration of DSPA for granting research approval and for assistance with obtaining permits; and the Primate Habituation 
390 Programme for providing logistical support in the field. We thank Luis Barriero, George Perry, and Laure Segurel for important comments on this manuscript, as well all of our field assistants and trackers for their help in the field. This work was supported by NSF grant 0935347, Grant Agency of the Czech Republic (number 206/09/0927) and funds from the University of Minnesota College of Biological Sciences. This publication

395 derives from the HPL-lab, Laboratory for Infectious Diseases Common to Humans and (non-Human) Primates, Czech Republic and was also co-financed by the European Social Fund and state budget of the Czech Republic. This work was supported in part by the University of Minnesota Supercomputing Institute.

The authors declare no conflict of interests. 
References

1. Turnbaugh PJ, et al (2007) The human microbiome project. Nature 449(7164): 804-810.

2. Human Microbiome Project Consortium (2012) Structure, function and diversity of the healthy human microbiome. Nature 486(7402): 207-214.

3. Ou J, et al (2013) Diet, microbiota, and microbial metabolites in colon cancer risk in rural africans and african americans. Am J Clin Nutr 98(1): 111-120.

4. Yatsunenko T, et al (2012) Human gut microbiome viewed across age and geography. Nature 486(7402): 222-227.

5. Obregon-Tito A, et al (2015) Subsistence strategies in traditional societies distinguish gut microbiomes. Nat Commun 6

6. Ley RE (2010) Obesity and the human microbiome. Curr Opin Gastroenterol 26(1): 5-11.

7. Bailey RC, et al (1989) Hunting and gathering in tropical rain forest: Is it possible?. American Anthropologist 91(1): 59-82.

8. Yamauchi T, Sato H \& Kawamura K (2014) Nutritional status and physical fitness of pygmy hunter-gatherers living in the african rainforests. African Study Monographs.Supplementary Issue. 47: 25-34.

9. Hart TB \& Hart JA (1986) The ecological basis of hunter-gatherer subsistence in african rain forests: The mbuti of eastern zaire. Hum Ecol 14(1): 29-55.

10. Perry GH, et al (2014) Adaptive, convergent origins of the pygmy phenotype in african rainforest hunter-gatherers. Proc Natl Acad Sci U S A 111(35): E3596-603.

11. Migliano AB, et al (2013) Evolution of the pygmy phenotype: Evidence of positive selection from genome-wide scans in african, asian, and melanesian pygmies. Human Biology 85(1): 251284.

12. Perry GH \& Dominy NJ (2009) Evolution of the human pygmy phenotype. Trends in Ecology \& Evolution 24(4): 218-225.

13. Schnorr SL, et al (2014) Gut microbiome of the hadza hunter-gatherers. Nat Commun 5: 3654 .

14. De Filippo C, et al (2010) Impact of diet in shaping gut microbiota revealed by a comparative study in children from europe and rural africa. Proc Natl Acad Sci U S A 107(33): 14691-14696.

15. O'Keefe SJD, et al (2015) Fat, fiber and colorectal cancer risk in african americans and rural africans. Nature Communications In press 
16. Sekimoto H, Shimada O, Makanishi M, Nakano T \& Katayama O (1983) Interrelationship between serum and fecal sterols. Jpn J Med 22(1): 14-20.

17. Okuda T \& Ito H (2011) Tannins of constant structure in medicinal and food plantshydrolyzable tannins and polyphenols related to tannins. Molecules 16(3): 2191-2217.

18. Weisburg WG, et al (1989) A phylogenetic analysis of the mycoplasmas: Basis for their classification. Journal of Bacteriology 171(12): 6455-6467.

19. Plugge CM \& Zoetendal EG (2014) in The Prokaryotes, (Springer, pp 1019-1021.

20. Wang Y, Li X, Mao Y \& Blaschek HP (2012) Genome-wide dynamic transcriptional profiling in clostridium beijerinckii NCIMB 8052 using single-nucleotide resolution RNA-seq. BMC Genomics 13: 102-2164-13-102.

21. Frey JC, et al (2006) Fecal bacterial diversity in a wild gorilla. Appl Environ Microbiol 72(5): 3788-3792.

22. Lin A, et al (2013) Distinct distal gut microbiome diversity and composition in healthy children from bangladesh and the united states. PloS One 8(1): e53838.

23. Azad MB, et al (2014) The gut microbiome and the hygiene hypothesis of allergic disease. impact of pets and siblings on infant gut microbiota. Annals of the American Thoracic Society 11(Supplement 1): S73-S73.

24. Nakazawa F, et al (2000) Description of mogibacterium pumilum gen. nov., sp. nov. and mogibacterium vescum gen. nov., sp. nov., and reclassification of eubacterium timidum (holdeman et al. 1980) as mogibacterium timidum gen. nov., comb. nov. Int J Syst Evol Microbiol 50 Pt 2: 679-688.

25. Cimprich RE \& Rooney JR (1977) Corynebacterium equi enteritis in foals. Veterinary Pathology Online 14(2): 95-102.

26. Willing BP, et al (2010) A pyrosequencing study in twins shows that gastrointestinal microbial profiles vary with inflammatory bowel disease phenotypes. Gastroenterology 139: 1844-1854.

27. Sakamoto M, et al (2009) Butyricimonas synergistica gen. nov., sp. nov. and butyricimonas virosa sp. nov., butyric acid-producing bacteria in the family 'porphyromonadaceae' isolated from rat faeces. Int J Syst Evol Microbiol 59(Pt 7): 1748-1753.

28. Wu GD, et al (2011) Linking long-term dietary patterns with gut microbial enterotypes. Science 333(6052): 105-108.

29. David LA, et al (2013) Diet rapidly and reproducibly alters the human gut microbiome. Nature

30. Langille MGI, et al (2013) Predictive functional profiling of microbial communities using 16S rRNA marker gene sequences. Nat Biotechnol 31(9): 814-21. 
31. Remis MJ \& Jost Robinson CA (2014) Examining short $\square$ term nutritional status among BaAka foragers in transitional economies. Am J Phys Anthropol 154(3): 365-375.

32. Milton K (1999) Nutritional characteristics of wild primate foods: Do the diets of our closest living relatives have lessons for us?. Nutrition 15(6): 488-498.

33. Bahuchet S, McKey D \& De Garine I (1991) Wild yams revisited: Is independence from agriculture possible for rain forest hunter-gatherers?. Hum Ecol 19(2): 213-243.

34. Yasuoka H (2013) Dense wild yam patches established by hunter-gatherer camps: Beyond the wild yam question, toward the historical ecology of rainforests. Hum Ecol 41(3): 465.

35. Yamauchi T, Sato H \& Kawamura K (2000) Nutritional status, activity pattern, and dietary intake among the baka hunter-gatherers in the village camps in cameroon. African Study Monographs 21(2): 67-82.

36. Flint HJ (2004) Polysaccharide breakdown by anaerobic microorganisms inhabiting the mammalian gut. Adv Appl Microbiol 56: 89-120.

37. Flint HJ, Bayer EA, Rincon MT, Lamed R \& White BA (2008) Polysaccharide utilization by gut bacteria: Potential for new insights from genomic analysis. Nature Reviews Microbiology 6(2): 121-131.

38. Chung K (1997) Gastrointestinal microbiology of monogastrics. Gastrointestinal Microbiology, eds Mackie R \& White B (Springer US), pp 511-582.

39. Pope PB, et al (2011) Isolation of succinivibrionaceae implicated in low methane emissions from tammar wallabies. Science 333(6042): 646-648.

40. Tito RY, et al (2012) Insights from characterizing extinct human gut microbiomes. PLoS One 7(12): e51146.

41. Uenishi G, et al (2007) Molecular analyses of the intestinal microbiota of chimpanzees in the wild and in captivity. Am J Primatol 69(4): 367-376.

42. Ochman H, et al (2010) Evolutionary relationships of wild hominids recapitulated by gut microbial communities. PLoS Biol 8(11): e1000546.

43. Cordain L, et al (2005) Origins and evolution of the western diet: Health implications for the 21st century. Am J Clin Nutr 81(2): 341-354.

44. Zhang X, et al (2013) Anti-hyperlipidemic effects and potential mechanisms of action of the caffeoylquinic acid-rich pandanus tectorius fruit extract in hamsters fed a high fat-diet. PloS One 8(4): e61922.

45. Cordain L, Eaton S, Brand Miller J, Mann N \& Hill K (2002) Original communications-the paradoxical nature of hunter-gatherer diets: Meat-based, yet non-atherogenic. Eur J Clin Nutr 56(1): S42. 
46. Devillard E, McIntosh FM, Duncan SH \& Wallace RJ (2007) Metabolism of linoleic acid by human gut bacteria: Different routes for biosynthesis of conjugated linoleic acid. Journal of Bacteriology 189(6): 2566-2570.

47. Daniel H, et al (2014) High-fat diet alters gut microbiota physiology in mice. ISME J 8(2): 295-308.

48. Hewlett BS (2014) Hunter-Gatherers of the Congo Basin: Cultures, Histories, and Biology of African Pygmies, (Transaction Publishers,

49. Claesson MJ, et al (2012) Gut microbiota composition correlates with diet and health in the elderly. Nature 488(7410): 178-184.

50. Round JL \& Mazmanian SK (2009) The gut microbiota shapes intestinal immune responses during health and disease. Nat Rev Immunol 9(5): 313-323.

51. Møller AP (1998) Evidence of larger impact of parasites on hosts in the tropics: Investment in immune function within and outside the tropics. Oikos : 265-270.

52. Bennett FJ, Kagan IG, Barnicot NA \& Woodburn JC (1970) Helminth and protozoal parasites of the hadza of tanzania. Trans R Soc Trop Med Hyg 64(6): 857-880.

53. Jarvis JP, et al (2012) Patterns of ancestry, signatures of natural selection, and genetic association with stature in western african pygmies. PLoS Genetics 8(4): e1002641.

54. Van den Abbeele P, Van de Wiele T, Verstraete W \& Possemiers S (2011) The host selects mucosal and luminal associations of coevolved gut microorganisms: A novel concept. FEMS Microbiol Rev 35(4): 681-704.

55. Shea BT \& Bailey RC (1996) Allometry and adaptation of body proportions and stature in african pygmies. Am J Phys Anthropol 100(3): 311-340.

56. Moeller AH, et al (2014) Rapid changes in the gut microbiome during human evolution. Proc Natl Acad Sci U S A 111(46): 16431-16435.

57. Schloss PD, Gevers D \& Westcott SL (2011) Reducing the effects of PCR amplification and sequencing artifacts on 16S rRNA-based studies. PloS One 6(12): e27310.

58. Wang Q, Garrity GM, Tiedje JM \& Cole JR (2007) Naive bayesian classifier for rapid assignment of rRNA sequences into the new bacterial taxonomy. Appl Environ Microbiol 73(16): $5261-5267$.

59. Caporaso JG, et al (2010) QIIME allows analysis of high-throughput community sequencing data. Nat Methods 7: 335-336.

60. R Core Team (2014) R core team (2014). R: A language and environment for statistical computing. R foundation for statistical computing, vienna, austria. URL http://Www.Rproject.org/. 
61. Nenadic O \& Greenacre M (2007) Correspondence analysis in R, with two- and threedimensional graphics: The ca package. Journal of Statistical Software 20(3)

62. Oksanen J, et al (2012) Vegan: Community ecology package. http://CRAN.Rproject.org/package $=$ veganR package version $2.0-5$

63. Roberts DW (2012) Labsdv: Ordination and multivariate analysis for ecology. http://Ecology.msu.montana.edu/labdsv/R

64. Dufrene M \& Legendre P (1997) Species assemblages and indicator species: The need for a flexible asymmetrical approach. Ecol Monogr 67(3): 345-366.

65. Revelle W (2014) Psych: Procedures for psychological, psychometric, and personality researchR package version 1.4.5

66. Giraudoux P (2014) Pgirmess: Data analysis in ecology. http://CRAN.Rproject.org/package $=$ gplotsR package version 1.5.9

\section{Figure legends}

Figure 1. Gut microbiome and metabolome composition in the BaAka. (a)Relative abundance of taxa at phyla and family level. (b)Relative abundance of metabolites according to broad and specific classifications. (c) Subnetwork view of gut microbiomemetabolome interactions in the BaAka. Green and yellow nodes represent bacterial taxa (at the family level ) and metabolites respectively. Edges represent Spearman correlation coeficients between metabolites and families ( $r h o>0.5$ ). Node size is proportional to the number of connections, and edge width represents strength of the correlation.

Figure 2. Comparison of the BaAka gut microbiome against that of the Hadza, USA americans, and italians. (a) Diversity indexes; rarefied richness, shannon diversity and phylogenetic diversity(1,000 reads per sample). Different letters denote significant differences $(P<0.05)$ according to Kruskal-Wallis (adjusted) pair-wise comparisons. (b) Pair-wise unweighted unifrac distances within (upper panel) and between groups (lower panel). All within and between pairwise distances are significant (Differneces in medians, permutation test $\mathrm{P}<0.001$ ) unless stated in the grapgh (N.S). (c) Principal coordinates analysis based on unweighted unifrac distances. Each symbol corresponds to the $16 \mathrm{~S}$ rRNA bacterial community composition in one stool sample.

Figure 3. Taxa (at family level), driving gut microbiome composition differences among BaAka, Hadza, Americans (HMP) and Italians. Correspondence analysis (CA) showing indicator taxa in each group, the short distance between each taxa point and symbols gives an estimate of the relative abundance of such taxa in a given sample. For this analysis only significant discriminators, based on indicartor taxa analysis were used (see Methods). Axes 1 and 3, explaining 13.2 and 7.4\% of the variation are shown separating the 4 groups. Axis $2(11.4 \%)$ does not show a separation between BaAka and Hadza (Figure S4). The abundance and significance of all indicator taxa within a given group was corroborated using q-values (Table S1) and visulaized in boxplots. (Figure S3). 
Figure 4. Predicted functional potential characterizing the gut microbiome of the BaAka, African and western groups. Functional predictions were made with PICRUSt. Different letters denote significant differences $(P<0.05)$ according to Kruskal-Wallis (adjusted) pair-wise comparisons. B=BaAka pygmies, H=Hadza, IT=Italians, US=US Americans. 


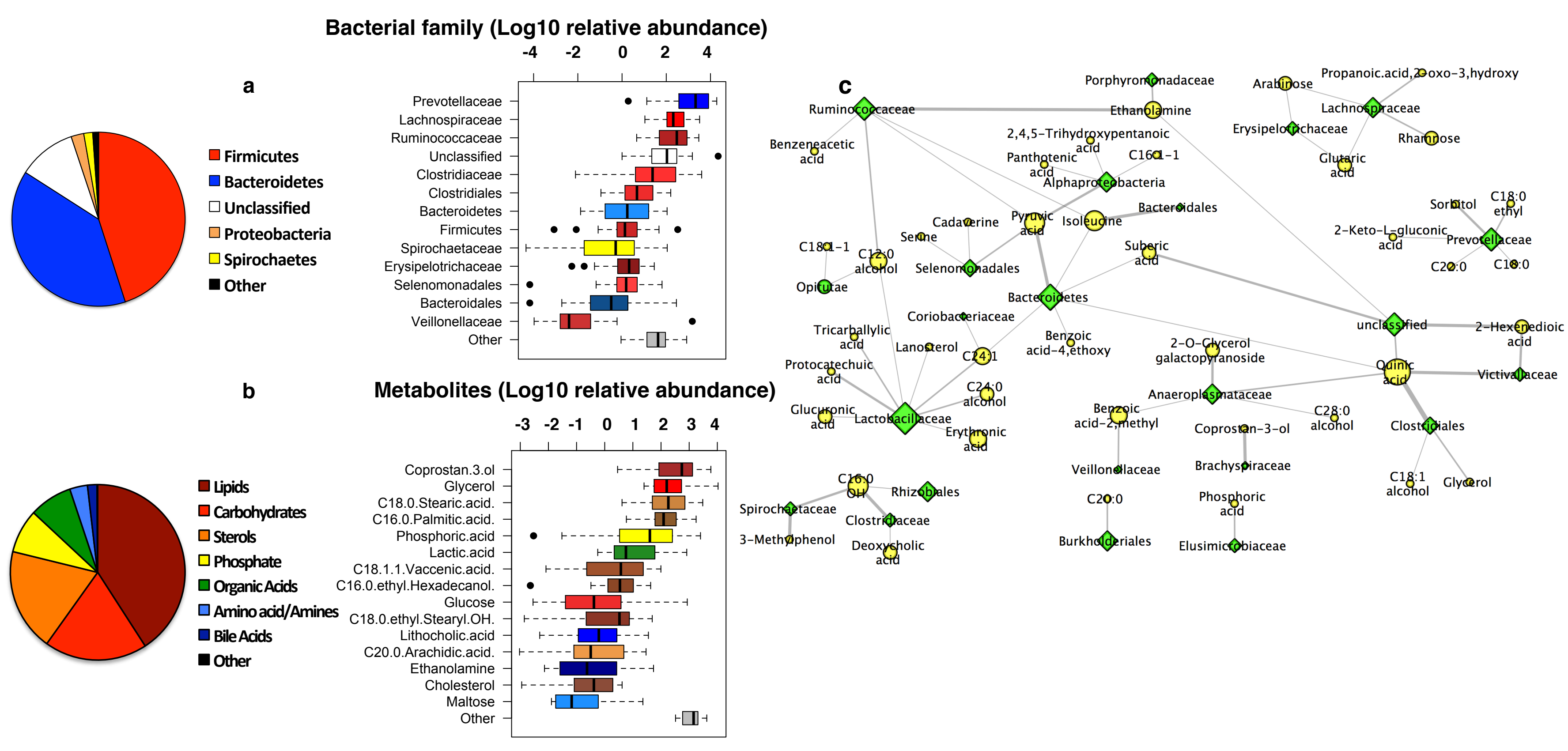




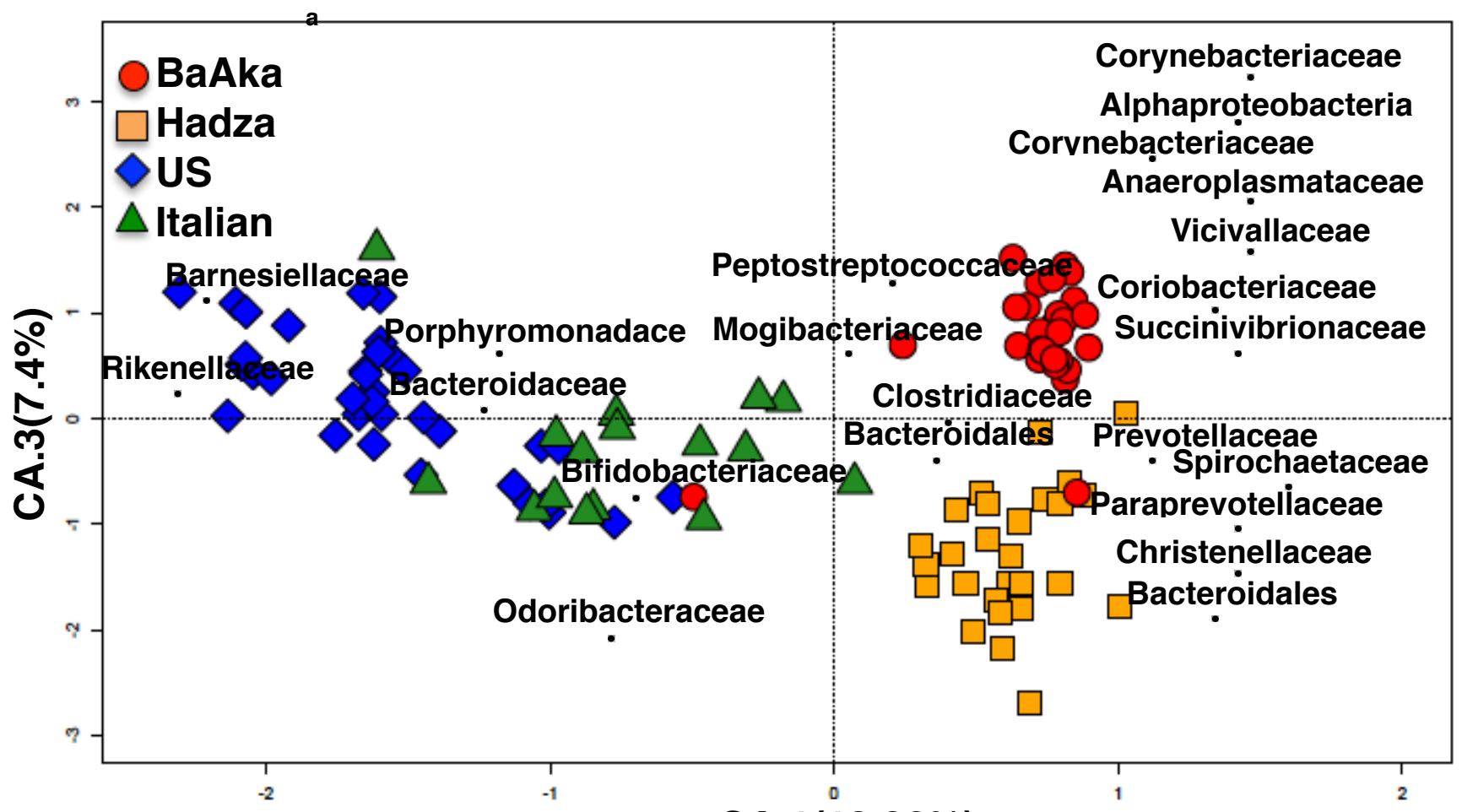

CA.1(13.26\%) 
Vibrio cholerae

pathogenic cycle (\%)

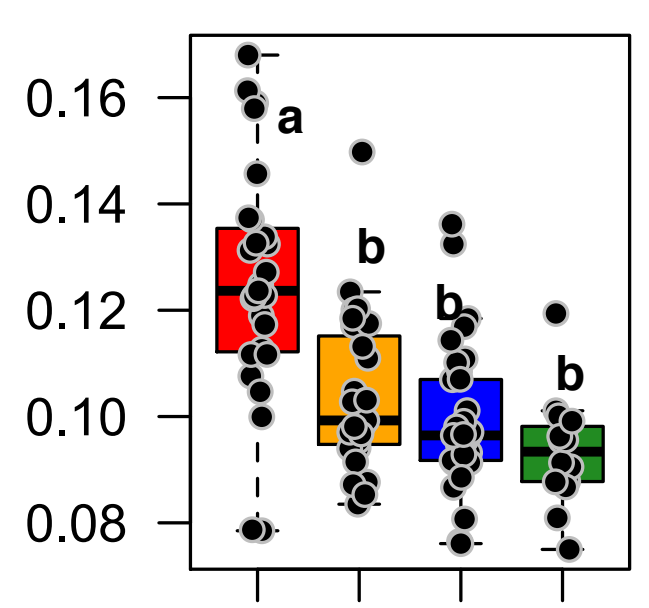

B $\quad$ H US IT
Staphylococcus aureus infection (\%)

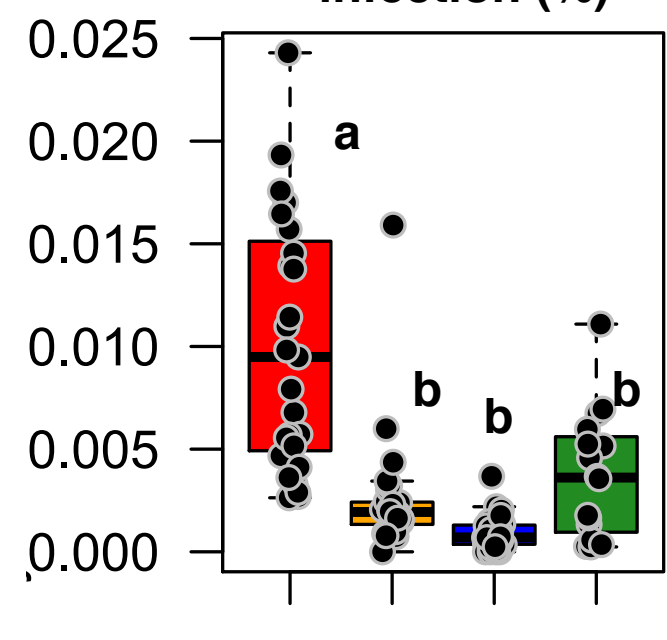

B H US IT
Pentose phosphate pathway (\%)

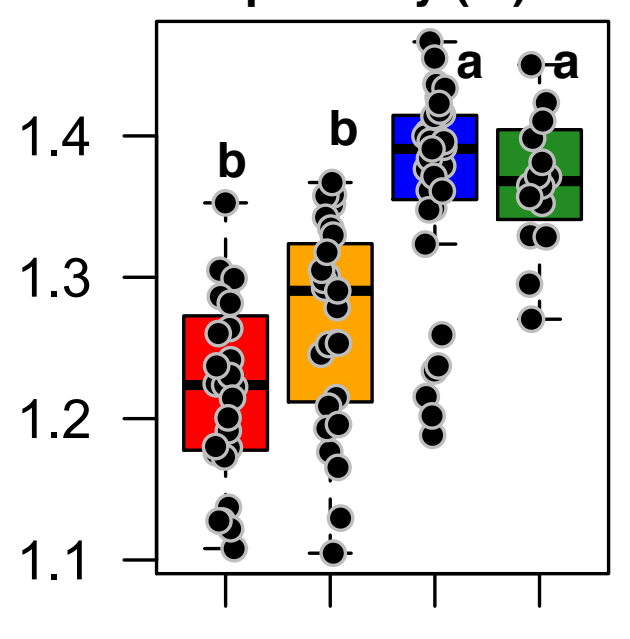

B H US IT
Galactose metabolism (\%)

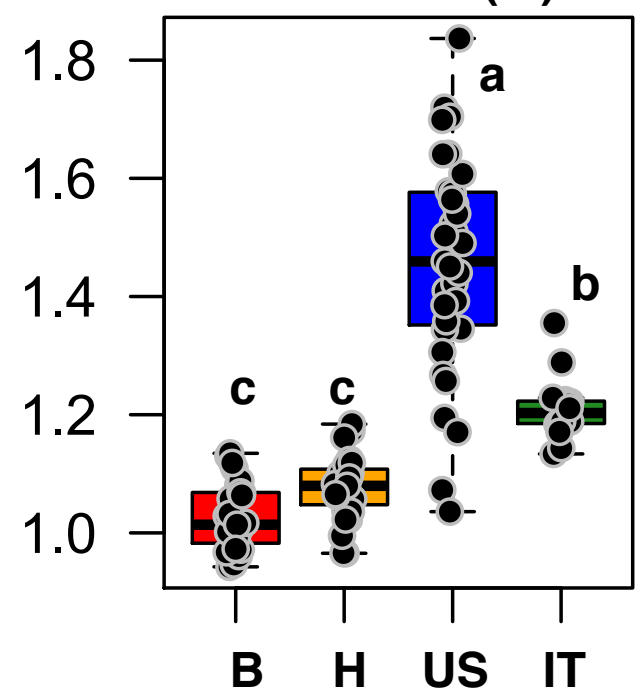

Starch and sucrose metabolism (\%)

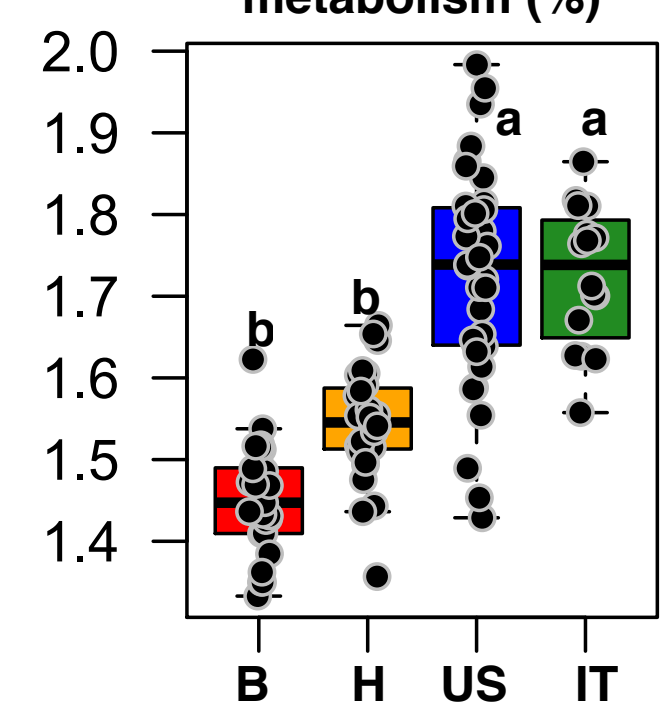

